\title{
A CONTRIBUIÇÃO DA LEITURA OROFACIAL NA COMUNICAÇÃO DO NEUROPATA AUDITIVO
}

\section{Lip reading contribution for the communication of the individual with auditory neuropathy}

\author{
Camila Paes Horacio $^{(1)}$, Maria Valéria Schmidt Goffi-Gomez ${ }^{(2)}$
}

\begin{abstract}
RESUMO
Objetivo: avaliar a contribuição da leitura orofacial na percepção de fala do neuropata auditivo. Métodos:foram incluídos neste estudo quatro indivíduos selecionados a partir dos seguintes critérios: pacientes adultos com neuropatia auditiva; idade superior a 18 anos e inferior a 60 anos; diagnóstico de neuropatia auditiva firmado pela equipe médica e nível mínimo de escolaridade (ser alfabetizado). Para a coleta de dados, foram realizadas as provas de percepção de fala sem e com leitura orofacial, que incluíram repetição de palavras dissilábicas e trissilábicas e repetição de frases em apresentação aberta (open-set). Resultados: verificou-se uma variação importante quanto ao grau da perda auditiva em todos os indivíduos e, em relação ao índice de reconhecimento de fala, observou-se discriminação variando de muito pobre a moderada. Observou-se que em todas as provas com apoio da leitura orofacial, houve melhora significativa do ponto de vista clínico da percepção da fala. Conclusão: o uso da leitura orofacial em provas específicas facilita a percepção auditiva, contribuindo para uma comunicação mais efetiva.
\end{abstract}

DESCRITORES: Doenças do Sistema Nervoso Periférico; Perda Auditiva; Percepção da Fala; Leitura Labial

\section{INTRODUÇÃO}

A comunicação humana é de extrema importância para a constituição, desenvolvimento e aprendizado do indivíduo. A surdez dificulta a comunicação, impedindo as relações entre os sons e as experiências vivenciadas, o desenvolvimento da função auditiva e a aquisição da linguagem ${ }^{1}$.

A neuropatia auditiva implica um acometimento da função neural do VIII par, com perda da sincronia na condução nervosa relacionada à alteração de mielinização dessas fibras, estando a função das células ciliadas ex-

(1) Fonoaudióloga Clínica; Prática Profissionalizante de longa duração (Colaboradora) - Hospital das Clínicas da Faculdade de Medicina da Universidade de São Paulo; Especialização em Motricidade Orofacial pelo Espaço de Fonoaudiologia Fernando Cordeiro; Pós-graduanda em Otorrinolaringologia pela Faculdade de Medicina da Universidade de São Paulo.

(2) Fonoaudióloga do Ambulatório de Otorrinolaringologia do Hospital das Clínicas da Faculdade de Medicina da Universidade de São Paulo; Doutora em Ciências dos Distúrbios da Comunicação Humana pela Universidade Federal de São Paulo. ternas preservada, enquanto a condução ao nervo e pelo nervo está prejudicada, podendo acontecer em qualquer idade ${ }^{2,3}$.

Os indivíduos com neuropatia auditiva podem apresentar características auditivas como: perda auditiva bilateral simétrica ou assimétrica, de leve a profunda, com reflexos ipsilateral e contralateral ausentes, dificuldade na discriminação vocal, desproporcional à configuração audiométrica e curva timpanométrica tipo $\mathrm{A}^{2-4}$.

Nas alterações retrococleares, observa-se aumento do limiar de reflexo tonal e/ou ausência dos limiares do reflexo estapediano ${ }^{5}$.

O conhecimento das características da deficiência auditiva e suas implicações podem influenciar na decisão da solução da recepção da mensagem, para resgatar o conteúdo com ênfase na leitura orofacial (LOF), no contexto, na atenção, na organização, no esclarecimento e no questio-namento, utilizando as estratégias de comunicação cognitivas $^{6}$.

Os indivíduos adultos com neuropatia auditiva têm alguma atenção aos sons ao seu redor, mas podem ser incapazes de realizar, de forma eficiente, a discriminação auditiva dos sons da fala; aqueles com resíduo audi- 
tivo ou com instalação tardia da neuropatia auditiva progressiva, dependem muito da LOF para complementar a informação auditiva ${ }^{2,7}$.

No entanto, na literatura, há poucos relatos de pesquisas realizadas com o intuito de investigar a contribuição da LOF na comunicação do neuropata auditivo.

Com base nessas considerações, o objetivo deste estudo é avaliar a contribuição da leitura orofacial na percepção de fala do neuropata auditivo.

\section{MÉTODOS}

Foram incluídos neste estudo quatro indivíduos, sendo dois do sexo feminino e dois do sexo masculino, entre 21 e 49 anos de idade, que preencheram os seguintes critérios da amostra: pacientes adultos com neuropatia auditiva; idade superior a 18 anos e inferior a 60 anos; diagnóstico de neuropatia auditiva firmado pela equipe médica e nível mínimo de escolaridade (ser alfabetizado), sem alterações neurológicas e/ou cogni-tivas. Todos os participantes deste estudo eram registrados e atendidos no Ambulatório de Otorrinolaringologia - Setor de Fonoaudiologia do Hospital das Clínicas da Faculdade de Medicina da Universidade de São Paulo.

O diagnóstico de neuropatia auditiva firmado pela equipe médica no prontuário e a avaliação audiológica básica (audiometria tonal e vocal, timpanometria e pesquisa de reflexos estape-dianos), foram realizados para verificar os aspectos audiológicos, caracterizando o grupo estudado. Todos os indivíduos haviam realizado treinamento de LOF antes da avaliação, por um período mínimo de cinco meses.

Para a coleta de dados, foram realizadas as provas de percepção de fala, sem e com LOF, que incluíram repetição de palavras dissilábicas ${ }^{8}$ e trissilábicas ${ }^{9}$ e repetição de frases ${ }^{10}$ em apresentação aberta (open-set).

No que se refere ao posicionamento do indivíduo, o mesmo encontrava-se sentado em frente à examinadora, a pelo menos um metro de distância, durante a situação de avaliação, que foi gravada para posterior análise e

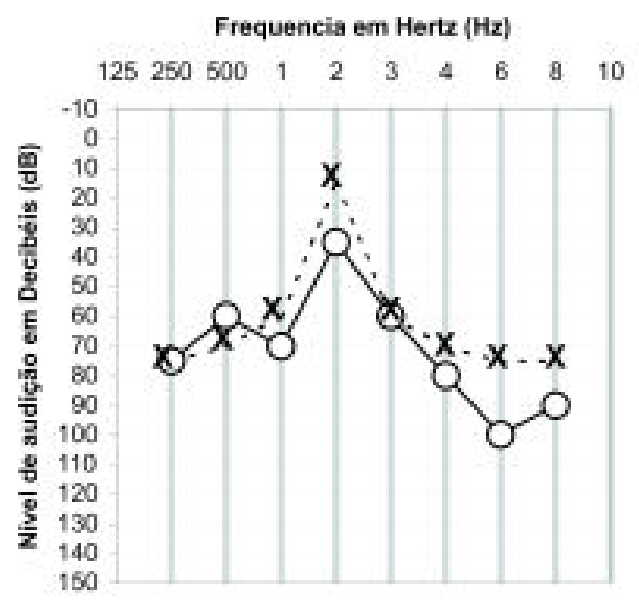

transcrição.

O presente estudo foi aprovado pela Comissão de Ética para análise de projetos de pesquisa - CAPPesq da Diretoria Clínica do Hospital das Clínicas e da FMUSP sob o protocolo $n^{\circ} 467 / 06$, sendo que todos os indivíduos da pesquisa concordaram com a sua realização e com a divulgação dos resultados, mediante a assinatura do Termo de Consentimento Informado.

Os dados coletados foram analisados qualitativamente, com ferramentas da estatística descritiva (média aritmética e desvio padrão).

\section{RESULTADOS}

Nas Figuras 1 a 4 são apresentados os dados relacionados ao audiograma e à timpanometria da cada indivíduo.

$\mathrm{Na}$ Tabela 1 são apresentados os resultados da audiometria tonal e vocal e os limiares dos reflexos estapedianos.

Quanto ao grau da perda auditiva, pode ser observada uma variação de grau leve a profundo e em relação à timpanometria, teve como resultado a curva do tipo A, tanto com ausência e/ou presença de reflexos estapedianos contralaterais. Verificou-se uma variação importante quanto ao grau da perda auditiva em todos os indivíduos. Em relação ao índice de reconhecimento de fala (IRF), observou-se discriminação variando de extremamente ruim a regular.

Na Tabela 2, estão descritos os resultados das provas de percepção de fala sem e com LOF. Verificou-se, na prova de repetição de dissílabos sem LOF, resultados variando de 0 a $48 \%$ e com LOF de 44 a $96 \%$; na prova de repetição de trissílabos, observou-se resultados sem LOF entre $4 \%$ a $84 \%$ e com LOF de $68 \%$ a $100 \%$.

Quanto à prova de repetição de frases, obtivemos na lista 1 sem LOF valores entre 0 e $80 \%$ e com LOF de $60 \%$ a $90 \%$, bem como na lista 3 , os resultados variaram entre 0 e $70 \%$ (sem LOF) e $80 \%$ a $100 \%$ (com LOF).

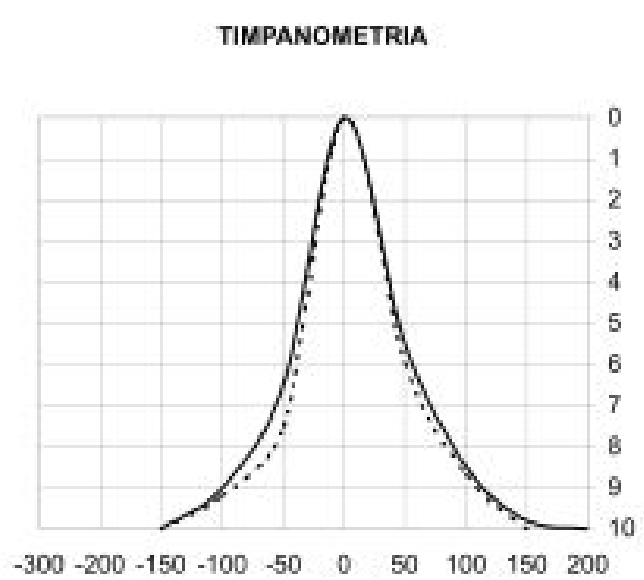

Figura 1 - Audiometria tonal e timpanometria do indivíduo 1 

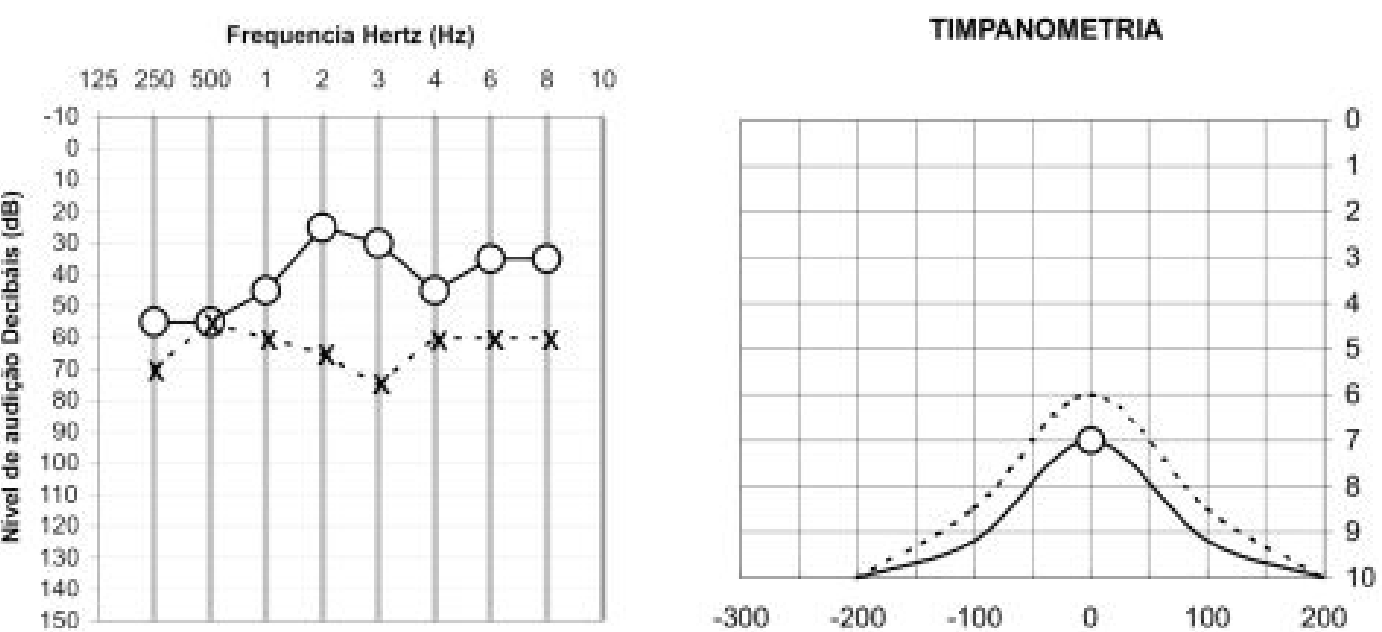

Figura 2 - Audiometria tonal e timpanometria do indivíduo 2

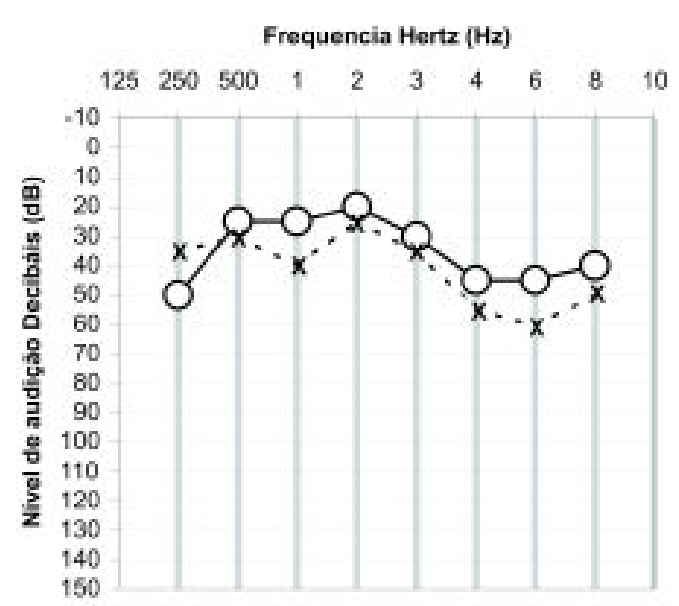

TIMPANOMETRIA

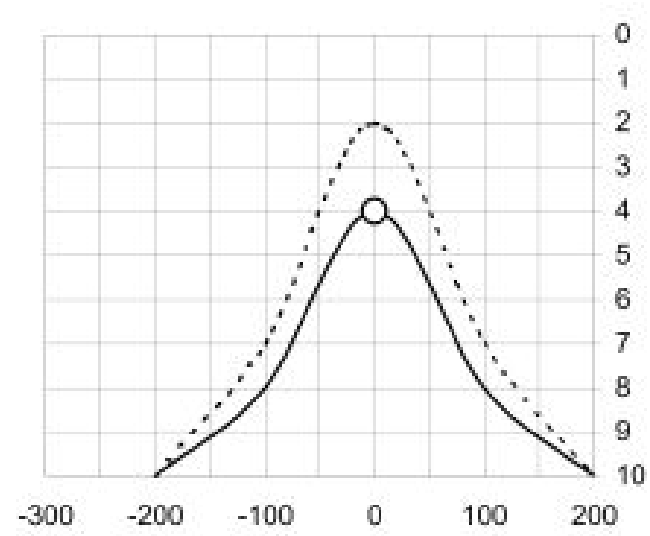

Figura 3 - Audiometria tonal e timpanometria do indivíduo 3
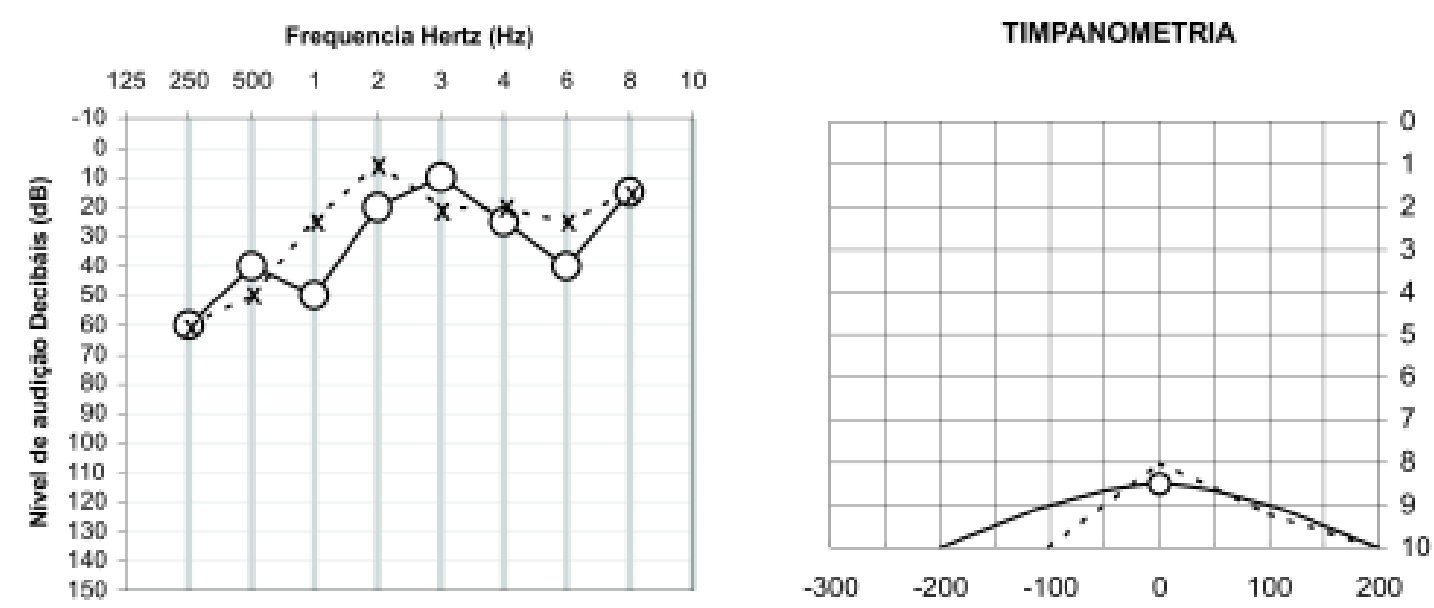

Figura 4 - Audiometria tonal e timpanometria do indivíduo 4 
Tabela 1 - Resultados da audiometria tonal e vocal, SRT (dBNA), IRF (\%) e dos limiares de reflexo estapediano (dBNA)

\begin{tabular}{|c|c|c|c|c|c|c|c|c|c|c|c|c|c|c|}
\hline \multirow[t]{2}{*}{ Indivíduo } & \multirow[t]{2}{*}{ Orelha } & \multicolumn{7}{|c|}{ Freqüência $(\mathrm{Hz})$} & \multirow[t]{2}{*}{ SRT } & \multirow[t]{2}{*}{ IRF (\%) } & \multicolumn{4}{|c|}{$\begin{array}{c}\text { Reflexo } \\
\text { estapediano }\end{array}$} \\
\hline & & 500 & $1 \mathrm{~K}$ & $2 \mathrm{~K}$ & $3 \mathrm{~K}$ & $4 \mathrm{~K}$ & $6 \mathrm{~K}$ & $8 \mathrm{~K}$ & & & 500 & $1 \mathrm{~K}$ & $2 \mathrm{~K}$ & $4 \mathrm{~K}$ \\
\hline \multirow{2}{*}{1} & OD & 60 & 70 & 40 & 60 & 80 & 100 & 90 & $25^{(\operatorname{LOV})}$ & - & - & - & - & - \\
\hline & OE & 70 & 60 & 15 & 60 & 70 & 75 & 75 & 25 & - & - & - & - & - \\
\hline \multirow[b]{2}{*}{2} & OD & 55 & 45 & 25 & 30 & 45 & 35 & 35 & 65 & $28 \% 95 \mathrm{~dB}$ & - & - & - & - \\
\hline & OE & 55 & 60 & 65 & 75 & 60 & 60 & 60 & 90 & $20 \% 100 \mathrm{~dB}$ & - & - & - & - \\
\hline & OD & 25 & 25 & 20 & 30 & 45 & 45 & 40 & 30 & $44 \% 70 \mathrm{~dB}$ & 105 & 120 & - & - \\
\hline & OE & 30 & 40 & 25 & 35 & 55 & 60 & 50 & 40 & $48 \% 70 \mathrm{~dB}$ & 100 & 105 & 115 & - \\
\hline & OD & 40 & 50 & 20 & 10 & 25 & 40 & 15 & 65 & $40 \% 80 \mathrm{~dB}$ & 40 & 120 & - & - \\
\hline & OE & 50 & 25 & 5 & 20 & 20 & 25 & 15 & 25 & $72 \% 55 \mathrm{~dB}$ & 50 & 120 & 120 & - \\
\hline
\end{tabular}

$S R T=$ limiar de recepção de fala; $I R F=$ índice de reconhecimento de fala; $O D=$ orelha direita; $O E=$ orelha esquerda; $d B$ NA= decibel Nível de Audição.

Tabela 2 - Resultados das provas de percepção de fala sem e com LOF (\%)

\begin{tabular}{|c|c|c|c|c|c|c|c|c|}
\hline \multirow{5}{*}{ Individuos } & \multicolumn{8}{|c|}{ Provas } \\
\hline & \multirow{2}{*}{\multicolumn{2}{|c|}{ Dissílabos (\%) }} & \multirow{2}{*}{\multicolumn{2}{|c|}{ Trissílabos (\%) }} & \multicolumn{4}{|c|}{ Frases (\%) } \\
\hline & & & & & \multicolumn{2}{|c|}{ Lista 1} & \multicolumn{2}{|c|}{ Lista 3} \\
\hline & Sem & Com & Sem & Com & Sem & Com & Sem & Com \\
\hline & LOF & LOF & LOF & LOF & LOF & LOF & LOF & LOF \\
\hline 1 & 0 & 44 & 4 & 68 & 0 & 60 & 0 & 80 \\
\hline 2 & 16 & 76 & 20 & 96 & 10 & 80 & 30 & 90 \\
\hline 3 & 48 & 80 & 52 & 84 & 10 & 70 & 30 & 100 \\
\hline 4 & 44 & 96 & 84 & 100 & 80 & 90 & 70 & 90 \\
\hline Média & 27 & 74 & 40 & 87 & 25 & 75 & 32,5 & 90 \\
\hline DV & 22,95 & 21,79 & 35,48 & 14,38 & 36,97 & 12,91 & 28,72 & 8,16 \\
\hline Mínimo & 0 & 44 & 4 & 68 & 0 & 60 & 0 & 80 \\
\hline Máximo & 48 & 96 & 84 & 100 & 80 & 90 & 70 & 100 \\
\hline $\begin{array}{l}\text { Contribuição } \\
\text { LOF }\end{array}$ & \multicolumn{2}{|c|}{47} & \multicolumn{2}{|c|}{47} & \multicolumn{2}{|c|}{50} & \multicolumn{2}{|c|}{57,5} \\
\hline
\end{tabular}

LOF = leitura orofacial; $\mathrm{DV}=$ desvio padrão 


\section{DISCUSSÃO}

A neuropatia auditiva é um acometimento da função neural do VIII par, com perda auditiva de graus variados, podendo ocorrer em qualquer idade ${ }^{2,3}$. Quando se fala em comunicação, remete-se à importância que ela exerce no desenvolvimento do indivíduo; logo, se há um comprometimento, as experiências, a função auditiva e a aquisição e desenvolvimento da fala e da linguagem, serão prejudicados ${ }^{1-3}$.

A partir dos dados apresentados nas Figuras 1 a 4 , verificou-se perda auditiva variando de grau leve a grave, ${ }^{11}$ nos indivíduos estudados com timpanometria do tipo $A$ e reflexos ausentes. De fato, a literatura ${ }^{2,3}$ mostra que os indivíduos com neuropatia auditiva podem apresentar perda auditiva bilateral simétrica ou assimétrica, de grau leve a profundo, bem como ausência e/ou reflexos estapedianos com limiares aumentados ${ }^{4,5}$.

Os reflexos estapedianos contralaterais, quando presentes, mostraram-se elevados, sugerindo alteração neural ${ }^{5}$. É possível que a falta de sincronia represente a alteração da atividade neural, responsável pelo disparo dos reflexos acústicos, ausente ou elevado ${ }^{12}$, conforme Tabela 1 , pois apesar desses indivíduos terem atenção aos sons, a falta da discriminação auditiva eficiente prejudica a compreensão da mensagem ${ }^{2}$.

Conforme os resultados apresentados na Tabela 1 , verificou-se que, além da perda auditiva, o índice de reconhecimento de fala (IRF) mostrou-se incompatível com o grau da perda auditiva em ambas as orelhas. Esses resultados confirmam que os indivíduos adultos com neuropatia auditiva têm dificuldade na discriminação vocal ${ }^{13}$.

Conforme os autores ${ }^{13}$ observaram, uma discriminação auditiva, variando de muito pobre (menor que $50 \%$ ) até discriminação moderada $(60 \%$ a $75 \%$ ), pode ocasionar uma incapacidade para acompanhar uma conversação.

A percepção de fala está relacionada com a com- preensão que o indivíduo realiza, a partir da mensagem auditiva; logo, aqueles que já desenvolveram a linguagem oral poderiam aperfeiçoar a percepção e a informação auditiva para o treinamento da LOF, pois o entendimento, a compreensão e a produção da fala propriamente dita, estão dentro de processos que têm como objetivo primordial a comunicação ${ }^{2,14}$.

De acordo com os dados apresentados na Tabela 2 , observou-se que em todas as provas com apoio da LOF, houve melhora clínica significativa da percepção da fala, facilitando assim a repetição, variando de $40 \%$ a $60 \%$ nas provas realizadas.

Os resultados apresentados (Tabela 2) corroboram as afirmações de que a percepção de fala, a informação auditiva, a recepção da mensagem e o conteúdo, são importantes no treinamento da LOF e que os indivíduos neuropatas auditivos dependem da LOF para completar a informação auditiva ${ }^{2,6,7}$.

Acredita-se que a leitura orofacial é uma ferramenta que pode ser utilizada com a maioria dos indivíduos com problemas de audição, bem como com os neuropatas auditivos. Quanto maiores forem a informação auditiva e o contexto, com apoio na pista visual, melhor será o desempenho comunicativo.

Considerando todos os aspectos abordados e analisados acima, pode-se observar que a leitura orofacial e o seu treinamento favorecem e contribuem para que a comunicação do neuropata seja mais efetiva.

Portanto, os achados deste estudo permitem incentivar a realização de novos trabalhos com o mesmo intuito, bem como a utilização de estratégias que englobem o uso da leitura orofacial, principalmente para a melhora da percepção de fala.

\section{CONCLUSÃO}

Por meio deste estudo, verificou-se que o uso da leitura orofacial em provas específicas facilita a percepção auditiva, contribuindo para uma comunicação mais efetiva do neuropata auditivo.

\section{ABSTRACT}

Purpose: to evaluate the contribution of lip reading for the recognition of speech in patients with auditory neuropathy. Methods: four subjects were included in the study according to the following criteria: adult patients with auditory neuropathy, with ages over eighteen and under sixty years; auditory neuropathy diagnosis given by the medical team and minimal literacy level. Speech perception tests were performed with and without lip reading, including dissyllabic and trissyllabic word recognition and open set sentence recognition. Results: there was an important variation regarding the level of hearing loss in all the subjects; and a discrimination varying from poor to moderate was noted in relation to the speech recognition index. We observed that in all the tests with lip reading, there was a significant improvement of speech perception. Conclusion: the use of lip reading in specific situations enhances hearing perception, contributing for a more effective communication.

KEYWORDS: Peripheral Nervous System Diseases; Hearing Loss; Speech Perception; Lipreading 


\section{REFERÊNCIAS}

1. Bento RF, Miniti A, Marone SM. Tratado de otologia. In: Bento RF, Miniti A, Marone SM. Doenças do ouvido interno. São Paulo: Edusp; 1998. p. 257-391.

2. Hood LJ. Auditory neuropathy: what is it and what can we do about it?. Hear J.1998; 51(8):11-8.

3. Spinelli M, Breuel ML, Silva CMS. Relato de caso: neuropatia auditiva: aspectos clínicos, diagnósticos e terapêuticos. Rev Bras Otorrinolaringol. 2001; 67(6):863-7.

4. Kumar UA, Jayaram MM. Prevalence and audiological characteristics in individuals with auditory neuropathy/auditory dys-synchrony. Int J Audiol. 2006; 45(6):360-6.

5. Goffi-Gomez MVS, Pedalini MEBP. Testes audiológicos para a identificação de alterações cocleares e retrococleares. In: Lopes Filho OC. Tratado de fonoaudiologia. São Paulo: Lovise; 2000. p. 127-47.

6. Bóechat EM. Ouvir sob o prisma da estratégia [mestrado]. São Paulo (SP): Pontifícia Universidade Católica de São Paulo; 1992.

7. Starr A, Picton TW, Sininger Y, Hood LJ, Berlin Cl. Auditory neuropathy. Brain. 1996; 119(3):741-53.

8. Harris RW, Goffi MVS, Pedalini MEB, Merrill A,
Gygi MA. Reconhecimento de palavras dissilábicas psicometricamente equivalentes no Português Brasileiro faladas por indivíduos do sexo masculino e do sexo feminino. Pró-Fono. 2001; 13(2):249-62.

9. Harris RW, Goffi MVS, Pedalini MEB, Merrill A, Gygi MA. Palavras trissilábicas psicometricamente equivalentes faladas por indivíduos do sexo feminino e masculino. Pró-Fono. 2001; 13(1):37-53.

10. Costa MJ, lorio MCM, Albernaz PLM. Desenvolvimento de um teste para avaliar a habilidade de reconhecer a fala no silêncio e no ruído. Pró-Fono. 2000; 12(2):9-16.

11. Audiometric Classification of hearing impairments. Bureau International Audiophonologie. [períodico online], 1996. Disponível em: URL: http: //www.biap.org/ biapanglais/rec021eng.htm

12. Carvallo RMM. Imitanciometria. In: Ferreira LP, BefiLopes D, Limongi SCO. Tratado de fonoaudiologia. São Paulo: Lovise; 2004. p. 569-84.

13. Jerger J, Speaks C, Trammell JL. A new approach to speech audiometry. J Speech Hear Disord. 1968; 33(4):318-28.

14. Ciello CA. Habilidades em consciência fonológica em crianças de 4 a 8 anos de idade. Pró-Fono. 2002; 14(3):301-12.
RECEBIDO EM: 27/04/2007

ACEITO EM: 19/07/2007

Endereço para correspondência:

Rua Leandro Dupret, 741

São Paulo - SP

CEP: $04025-013$

Tel: (11) 50850004 Fax: (11) 50822235

E-mail: fonocamila@ig.com.br 